\title{
Empirical plasmapause models from magnetic indices
}

\author{
T. P. O'Brien \\ Space Science Department, Aerospace Corporation, El Segundo, California, USA
}

\author{
M. B. Moldwin \\ Institute of Geophysics and Planetary Physics, University of California, Los Angeles, California, USA \\ Received 30 July 2002; revised 5 September 2002; accepted 20 January 2003; published 18 February 2003.
}

[1] We use a database of CRRES in situ observations of plasmapause crossings to build empirical models of the plasmapause location as a function of geomagnetic indices. Previous models used the maximum value in $K p$ during the hours to days leading up to the plasmapause crossing. We find that a recent maximum in $A E$ or minimum in Dst provides a better model of the plasmapause radius than does maximum $K p$. $A E$ and Dst measure specific current systems (the auroral electrojet and ring current, respectively). The AE model suggests that substorms may be involved in the erosion of the plasmapause. The Dst model suggests that ring current may be formed by the same electric field that erodes the plasmapause. In more complex models, $K p$ and $A E$ can describe local time structure in the plasmapause, with the furthest plasmapause before dawn at quiet times, premidnight at active times. INDEX TERMS: 2768 Magnetospheric Physics: Plasmasphere; 2760 Magnetospheric Physics: Plasma convection; 2740 Magnetospheric Physics: Magnetospheric configuration and dynamics; 2712 Magnetospheric Physics: Electric fields (2411); 2730 Magnetospheric Physics: Magnetosphere-inner. Citation: O'Brien, T. P., and M. B. Moldwin, Empirical plasmapause models from magnetic indices, Geophys. Res. Lett., 30(4), 1152, doi:10.1029/2002GL016007, 2003.

\section{Introduction}

[2] The plasmapause represents the outer boundary of the plasmasphere, a region in the inner magnetosphere that is filled with trapped, cold, and dense plasma, whose motion is dominated by large-scale electric fields. Because the characteristics of the plasma change abruptly at the plasmapause, so do the characteristics of waves and other electromagnetic phenomena. Together, the plasma and wave characteristics inside and outside the plasmapause can influence a variety of other inner magnetospheric phenomena, including the ring current [Kozyra et al., 1995] and radiation belts [Horne and Thorne, 1998; Lorentzen et al., 2001]. Therefore, it is often necessary to know the time dependent location of the plasmapause, in order to constrain the dynamics of other local phenomena. During quiet times, the plasmasphere is thought to expand as higher altitude flux tubes slowly fill with plasma; during active times magnetospheric convection strips off the outer layers of the plasmasphere, bringing the plasmapause closer to the Earth [see, for example, Carpenter and Anderson, 1992].

Copyright 2003 by the American Geophysical Union. 0094-8276/03/2002GL016007\$05.00
[3] We follow the work of previous authors in modeling the plasmapause from ground-based geomagnetic indices [Carpenter and Anderson, 1992; Moldwin et al., 2002]. The location of the plasmapause is usually identified by a sharp radial gradient in the local electron density measured in situ by a spacecraft passing across the boundary. Previous authors have modeled the location using the maximum of $K p$ over an interval of several hours to a day prior to the plasmapause crossing. $K p$ has been used because it can be loosely related to the strength of convection [Volland, 1979], which nominally controls the location of the plasmapause [Nishida, 1966; Chappell et al., 1970].

[4] Recent research has highlighted specific phenomena that are likely to affect the plasmapause location. For example, Ridley and Liemohn [2002] have associated Dst, the rate of change in $D s t$, and the asymmetric ring current with strong enhancements of the inner magnetospheric electric field. Also, Goldstein et al. [2002] have shown examples from the IMAGE spacecraft of rapid contraction of the plasmapause owing to strong electric fields.

[5] Because $K p$ is only loosely associated with any particular physical manifestation, we have expanded the search for empirical plasmapause models to those geomagnetic indices that can be more immediately associated with magnetospheric phenomena. We develop models of the plasmapause location using $K p$ and other geomagnetic indices, including $A E, D s t$, and the asymmetry index $A S Y$. Unlike $K p$, these latter indices are (at least nominally) associated with specific current systems: $A E$, and its components, $A L$ and $A U$, describe the high latitude (outer magnetosphere) current systems, which respond to both global convection and substorm activity. Dst and $A S Y$ nominally measure the average and asymmetry of the equatorial currents flowing in the inner magnetosphere, which are thought to respond primarily to convection. We show that $A E$ and $D s t$ can be used to develop plasmapause models that are superior to those built on $K p$.

\section{Data}

[6] We use the database of over 900 plasmapause crossings developed by Moldwin et al. [2002] from in situ CRRES electron density observations made in 19901991. The plasmapause is defined as the inner-most sharp radial density gradient measured along the nearly-equatorial CRRES orbit. We use several geomagnetic indices, each of which measures slightly different characteristics of magnetic activity. $K p$ is a pseudo-logarithmic measure of the global geomagnetic disturbance level, and is provided by the National Geophysical Data Center. Dst and ASY reflect 
Table 1. Correlation of $L_{\mathrm{pp}}$ With Various Indicators

\begin{tabular}{lcrc}
\hline Indicator & $t_{1}$ & $t_{2}$ & $|\mathrm{ROCC}|$ \\
\hline$D s t$ & -24 & 0 & $0.67 \pm 0.02$ \\
$D s t$ & -12 & 0 & $0.67 \pm 0.02$ \\
$D s t$ & -24 & -1 & $0.67 \pm 0.02$ \\
$D s t$ & -36 & 0 & $0.66 \pm 0.02$ \\
$A E$ & -36 & 0 & $0.66 \pm 0.02$ \\
$A E$ & -36 & -1 & $0.66 \pm 0.02$ \\
$A E$ & -24 & 0 & $0.65 \pm 0.02$ \\
$A U$ & -36 & 0 & $0.65 \pm 0.02$ \\
$K p$ & -36 & -2 & $0.65 \pm 0.02$ \\
$K p$ & -24 & -2 & $0.64 \pm 0.02$ \\
$A L$ & -36 & 0 & $0.63 \pm 0.02$ \\
$A S Y$ & -36 & 0 & $0.63 \pm 0.03$ \\
$\Delta D s t$ & -72 & -2 & $0.50 \pm 0.03$ \\
\hline
\end{tabular}

the strength of the symmetric and asymmetric components of the equatorial ring current. We have used hourly 4-station $D s t$ and 1-minute 6-station $A S Y$, provided by Kyoto World Data Center. The auroral indices, $A E, A L$, and $A U$ measure the total, westward, and eastward electroject currents, respectively. We have used 1-minute provisional auroral indices provided by Kyoto World Data Center. For the definition of ASY, see Kawasaki and Akasofu [1971]; for all other indices, see Mayaud [1980].

\section{Correlation Analysis}

[7] Our first task is to identify useful indicators of the plasmapause location. As described above, we have chosen several candidates based on their association with various components of magnetospheric electric fields. We use a rank order correlation coefficient to evaluate each index of geomagnetic activity as a possible indicator of plasmapause location. We correlate the plasmapause location $\left(L_{\mathrm{pp}}\right)$ with running maxima of $K p, A E, A U$, and $A S Y$, and with running minima of $A L, D s t$, and $\Delta D s t$. In each case we vary the start $\left(t_{1}\right)$ and end $\left(t_{2}\right)$, in hours relative to the plasmapause crossing, of the interval over which the maximum or minimum is taken. We systematically explore a range of $t_{1}$ and $t_{2}$ values. Since the plasmapause is expected to take a day or more to recover, we vary $t_{1}$ from 0 to -72 hours. The plasmapause is believed to take several hours to respond to a change in convection; so, we vary $t_{2}$ from 0 to -6 hours, always maintaining $t_{1} \leq t_{2}$.

[8] Because the relationship between $L_{\mathrm{pp}}$ and any one of the candidate indicators maybe nonlinear, and because some of the quantities may have highly skewed distributions, we use the rank order correlation coefficient (ROCC) rather than the linear correlation coefficient. Whereas the linear correlation coefficient measures the quality of the optimal linear relationship between two variables, the ROCC measures the quality of the optimal nonlinear relationship, without specifying that relationship a priori. As with the linear correlation coefficient, 1 indicates perfect correlation, -1 indicates anticorrelation, and 0 indicates no correlation [Press et al., 1992].

[9] Table 1 provides some samples of correlations of $L_{\mathrm{pp}}$ with various indicators, including the best indicator built from each geomagnetic index. The best correlation was obtained for minimum $D s t$ taken over various combinations of the 24 hours preceding the CRRES plasmapause crossing. However, indicators built on $A E, A U$, and $K p$ per- formed similarly well (the uncertainties are given as standard errors: any values within 2 standard errors of each other are statistically indistinguishable at the $95 \%$ confidence level). Also, the correlation does not deteriorate significantly when the end time is adjusted by an hour or two. Therefore, the plasmapause can be modeled with similar accuracy using a variety of geomagnetic indices. The $A L$ and $A S Y$ correlations are probably worse than the $D s t$ correlation, and the $\Delta D s t$ correlation is significantly worse.

\section{Best-Fit Models}

[10] Having identified the indicators that are best correlated with the plasmapause location, we move on to develop simple quantitative models. We begin with the linear form

$$
L_{\mathrm{pp}}=a Q+b,
$$

where $Q$ is a representation of one of the indicators identified in the previous section: $Q=\max _{-36-2} K p, Q=$ $\log _{10} \max _{-36,0} A E$, or $Q=\log _{10}\left|\min _{-24,0} D s t\right|$. The notation $\max _{t_{1}, t_{2}} X$ indicates the maximum (or minimum) of $X$ taken from $t_{1}$ to $t_{2}$ hours relative to the plasmapause crossing. Figure 1 depicts the quality of each fit. Each panel shows considerable scatter, but the overall trend is obvious.

[11] Table 2 lists the fit coefficients from equation (1) and the RMS errors for all local times and for 4 6-hour local time sectors. The reader should note that the equation given
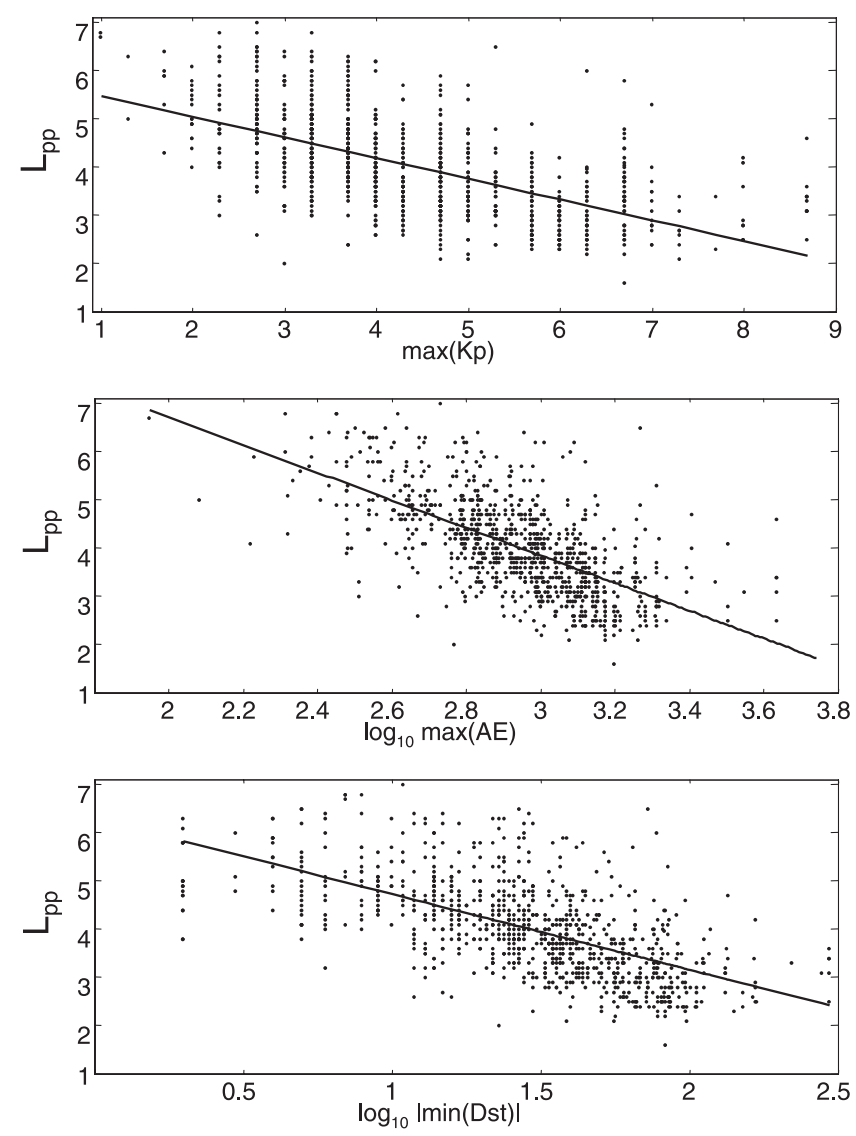

Figure 1. Panels (a)-(c) depict the fits of plasmapause location versus 3 geomagnetic indicators (see text). 
Table 2. Quality of 2-Parameter Fits to $L_{\mathrm{pp}}$

\begin{tabular}{|c|c|c|c|c|c|c|c|}
\hline & \multicolumn{2}{|c|}{ Fit } & \multicolumn{5}{|c|}{ RMSE } \\
\hline & $a$ & b & All & Night & Dawn & Day & Dusk \\
\hline & $-0.43 \pm 0.02$ & $5.9 \pm 0.1$ & 0.79 & 0.78 & 0.71 & 0.68 & 0.93 \\
\hline$A E$ & $-2.86 \pm 0.11$ & $12.4 \pm 0.3$ & 0.76 & 0.74 & 0.67 & 0.67 & 0.92 \\
\hline Dst & $-1.57 \pm 0.06$ & $6.3 \pm 0.1$ & 0.76 & 0.80 & 0.68 & 0.64 & 0.87 \\
\hline
\end{tabular}

in (1) is not necessarily the optimal model implied by the ROCCs of the correlation analysis above, and so some reordering of the fit quality is expected. The linear fit coefficients for the $K p$ model are similar to those given by Carpenter and Anderson [1992] $(a=-0.46, b=5.6)$ and Moldwin et al. [2002] $(a=-0.382 \pm 0.019, b=5.39 \pm$ $0.072)$. The slight discrepancies with our values $(a=-0.43$ $\pm 0.02, b=5.9 \pm 0.1)$ arise because of different $t_{1}$ and $t_{2}$ used in each study; also Carpenter and Anderson used plasmapause crossings only in the range $00-15$ hours local time. Our RMS errors are approximately $0.7-0.9 \mathrm{~L}$ in all local times taken together or in sectors. Based on bootstrap (Monte Carlo) confidence intervals [Press et al., 1992] in any column, all of the RMS errors reported in Table 2 are statistically indistinguishable, with the exception of the night sector, where the $A E$ model's RMS error is significantly lower than that of $D s t$. It is worth noting that $A E$ may provide a slightly superior model in the dawn sector as well, whereas Dst may provide a superior model in the day and dusk sectors.

\section{Local Time Analysis}

[12] In keeping with previous studies, we proceed from a simple $L_{\mathrm{pp}}$ model to a more complex model that describes the local time dependence of the plasmapause location. We increase the complexity of our fit to include a first harmonic in magnetic local time $(m l t)$ :

$$
\begin{gathered}
\{\phi=2 \pi(m l t / 24), \\
L_{\mathrm{pp}}=a_{1}\left[1+a_{m l t} \cos \left(\phi-a_{\phi}\right)\right] Q+b_{1}\left[1+b_{m l t} \cos \left(\phi-b_{\phi}\right)\right] .
\end{gathered}
$$

This equation can parsimoniously approximate a bulge, but does not assume one. This flexibility is appropriate, given the observation by Carpenter and Anderson [1992] that the plasmapause bulge is not necessarily evident in all schemes of plasmapause identification.

[13] The parameters of (3) have physical meaning: $a_{m l t}$ and $b_{m l t}$ provide the relative amplitude of the local time variation, while $a_{\phi}$ and $b_{\phi}$ provide the phase, i.e. the location of the bulge. Table 3 gives the best-fit coefficients of (3) for each model, as well as RMS errors as in Table 2. Examining $a_{m l t}$ and $b_{m l t}$, we see that the local time variation

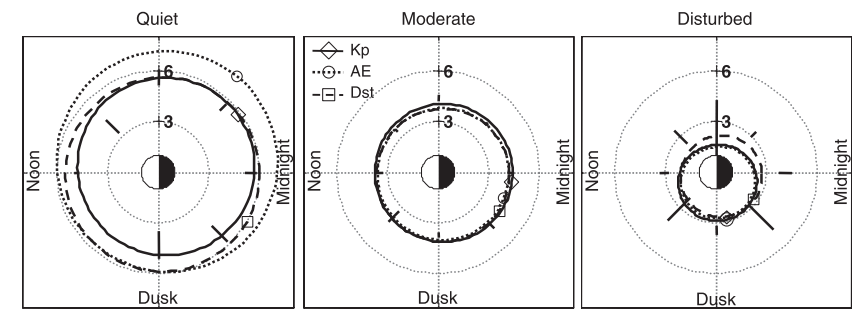

Figure 2. Each panel depicts the plasmapause shape $\left(L_{\mathrm{pp}}\right.$ in $R_{E}$ ) for three models. The symbols indicate the local time of maximum $L_{\mathrm{pp}}$ for each model. Thick radial lines indicate 2 standard deviations around the mean $L_{\mathrm{pp}}$ from CRRES measurements (see text). Magnetic activity increases from left to right.

in the Dst model is not statistically significant. Also, $b_{m l t}$ is not significant in the $K p$ model, indicating that the only part of the local time variation that is significant is the part modulated by $K p$. Judging from $a_{\phi}$ and $b_{\phi}$, the $K p$ and $A E$ models provide their maximum $L_{\mathrm{pp}}$ at either $\sim 1600$ or $\sim 0400$ magnetic local time, depending on the level of magnetic activity. The Dst model always gives a maximum $L_{\mathrm{pp}}$ at around 2100 mlt.

[14] We can examine the local time dependence further by simulating the plasmapause shape from each model for three levels of magnetic activity. We have identified these levels as the minimum (quiet, $K p=1, A E=89 \mathrm{nT}, D s t=$ $-2 \mathrm{nT}$ ), median (moderate, $K p=4, A E=914 \mathrm{nT}$, $D s t=$ $-30 \mathrm{nT}$ ), and maximum (disturbed, $K p=9^{-}, A E=4351$ $\mathrm{nT}, D s t=-298 \mathrm{nT}$ ) values of $Q$ for each model. Figure 2 depicts $L_{\mathrm{pp}}(\mathrm{mlt})$ for each model for the three levels of activity. Also, thick radial lines indicate 2 standard deviations around the mean $L_{\mathrm{pp}}$ from CRRES measurements in 3-hour local time bins, where the activity limits are defined by $Q=\max _{-36,-2} K p: Q_{\text {quiet }} \leq 2^{+}, 3^{-} \leq Q_{\text {moderate }}$ $\leq 6+$, and $Q_{\text {disturbed }} \geq 7^{-}$. At quiet times, the plasmapause is enlarged, with the $K p$ and $A E$ models placing the maximum $L_{\mathrm{pp}}$ before dawn. As we will see, the Dst model does not resolve much local time structure, and therefore has its maximum $L_{\mathrm{pp}}$ at a different location (premidnight). The predawn maximum $L_{\mathrm{pp}}$ reproduces well-established results [Carpenter and Anderson, 1992; Moldwin et al., 2002]. As activity increases, however, the plasmapause contracts, and the maximum rotates earlier to dusk (for the $K p$ and $A E$ models). The binned CRRES measurements give qualitatively similar results; because the quiet and disturbed models are describing more extreme conditions than are the binned CRRES data, the model curves are outside (inside) the CRRES averages for the quiet (disturbed) conditions.

[15] Finally, we examine the RMS errors for the three local-time models. As before, Table 3 reports the errors for

\begin{tabular}{|c|c|c|c|c|c|c|c|c|c|c|c|}
\hline & \multicolumn{6}{|c|}{ Fit } & \multicolumn{5}{|c|}{ RMSE } \\
\hline & $a_{1}$ & $a_{m l t}$ & $(24 / 2 \pi) a_{\phi}$ & $b_{1}$ & $b_{m l t}$ & $(24 / 2 \pi) b_{\phi}$ & All & Night & Dawn & Day & Dusk \\
\hline$K p$ & $-0.39 \pm 0.02$ & $-0.34 \pm 0.05$ & $16.6 \pm 0.2$ & $5.6 \pm 0.1$ & $0.12 \pm 0.17$ & $3 \pm 1$ & 0.74 & 0.76 & 0.69 & 0.57 & 0.88 \\
\hline$A E$ & $-2.60 \pm 0.12$ & $-0.30 \pm 0.21$ & $16.8 \pm 0.3$ & $11.6 \pm 0.4$ & $0.20 \pm 0.63$ & $4 \pm 1$ & 0.72 & 0.72 & 0.65 & 0.57 & 0.87 \\
\hline Dst & $-1.54 \pm 0.07$ & $-0.04 \pm 0.14$ & $20.6 \pm 2.3$ & $6.2 \pm 0.1$ & $0.04 \pm 0.20$ & $22 \pm 3$ & 0.73 & 0.76 & 0.67 & 0.53 & 0.85 \\
\hline
\end{tabular}

Table 3. Quality of 2-Parameter Fits to $L_{\mathrm{pp}}$ 
all local times and for 4 local time sectors. The RMS errors are generally lower than those for the simpler models, given in Table 2. Compared to the simpler models, when all local times are considered or when only the daytime sector is considered, the reduction in the RMS errors is significant at the $95 \%$ confidence level. Within each column of Table 3 , the RMS errors are statistically indistinguishable at the $95 \%$ confidence level. Nonetheless, as before, the $A E$ model performs best in the night and dawn sectors, whereas Dst performs best in the day and dusk sectors.

\section{Discussion}

[16] In general, our results confirmed those of previous researchers [Carpenter and Anderson, 1992; Moldwin et $a l ., 2002]$, and we have shown that, in addition to $K p$, other quantities, such as $D s t$ and $A E$ can be used to build a quantitative model of the plasmapause location. There is little statistical degradation in the quality of the plasmapause models built when the underlying geomagnetic index is replaced or when the start and ending intervals for the maximum or minimum are changed. For quiet times, our model predicts a plasmapause bulge on the dawn side. In the models built on $A E$ and $K p$, the bulge rotates through midnight, toward dusk as activity increases. This rotation may be attributable to a stronger and faster plasmapause response on the night side, where magnetic activity, such as convection and substorm injections, is also stronger.

[17] The success of the $A E$ model, especially relative to the $A L$ and $A U$ models, suggests that both convective and substorm electric fields are important for determining the plasmapause location. The $A E$ model is particularly effective in the night and dawn sectors. The poor performance of the $A S Y$ and $\triangle D$ st models is surprising, given the results of Ridley and Liemohn [2002], which relate both of these quantities to the inner magnetospheric electric field. It is therefore likely that these electric fields are associated with the fast relative motion seen by Goldstein et al. [2002], rather than with the absolute position of the plasmapause. Future statistical and case studies using high time resolution IMAGE plasmapause measurements will likely help test this association.

[18] The success of the minimum Dst model for the plasmapause location suggests that minimum Dst is a better proxy for the maximum strength of the convection electric field than is $K p$. Additionally, the lack of local time dependence in the Dst model indicates a possible role for ring current plasma in quickly eroding the plasmapause through non-convective processes. The Dst model can be used in ring current simulations because it relies primarily on past values of Dst. The Dst model also hints that loss of energetic electrons interior to or acceleration exterior to the plasmapause may be associated with the relationship between the peak in $L$ of energetic electron flux and minimum Dst observed by Tverskaya [1986].
[19] We note that our study is built upon the same dataset as that of Moldwin et al. [2002]. Our results, therefore, suffer from the same difficulties, including a possible bias to low $L_{\mathrm{pp}}$ on the day side due to the strict density gradient criteria used in identifying the plasmapause, and an orbital upper limit on $L_{\mathrm{pp}}$ of around 7. Nonetheless, the models we have produced demonstrate that $K p$ is not the only geomagnetic index that can be used successfully to model the plasmapause location.

[20] Acknowledgments. This project was supported by NASA grants NAG5-10972, NAGW-5153, and NAG5-4897. We acknowledge the CRRES plasma wave instrument (R.R. Anderson, PI), the Kyoto World Data Center (WDC C-2) for geomagnetism and the observatories that produce $A E$ and Dst, and the National Geophysical Data Center and the observatories that produce $K p$.

\section{References}

Carpenter, D. L., and R. R. Anderson, An ISEE/whistler model of equatorial electron density in the magnetosphere, J. Geophys. Res., 97, 10971108, 1992.

Chappell, C. R., K. K. Harris, and G. W. Sharp, A study of the influence of magnetic activity on the location of the plasmapause as measured by OGO 5, J. Geophys. Res., 75, 50-56, 1970.

Goldstein, J., R. A. Wolf, B. R. Sandel, W. T. Forrester, D. L. Gallager, P. H. Reiff, and R. Spiro, Rapid response of the plasmasphere to changes in the solar wind and IMF: Global plasmapause electric field measurements by IMAGE EUV, and simulation with the Rice MSM, Eos Trans. AGU, 83(19), Spring Meet. Suppl., SM41B-09, 2002.

Horne, R. B., and R. M. Thorne, Potential waves for relativistic electron scattering and stochastic acceleration during magnetic storms, Geophys. Res. Lett., 25, 3011-3014, 1998.

Kawasaki, K., and S.-I. Akasofu, Low-latitude DS component of geomagnetic storm field, J. Geophys. Res., 76, 2396-2405, 1971.

Kozyra, J. U., C. E. Rasmussen, R. H. Miller, and E. Villalon, Interaction of ring current and radiation belt protons with ducted plasmaspheric hiss, 2, Time evolution of the distribution function, J. Geophys. Res., 100, 21,911-21,920, 1995 .

Lorentzen, K. R., J. B. Blake, U. S. Inan, and J. Bortnik, Observations of relativistic electron microbursts in association with VLF chorus, J. Geophys. Res., 106, 6017-6027, 2001.

Mayaud, P. N. (Ed.), Derivation, Meaning and Use of Geomagnetic Indices, Geophys. Monogr. Ser., vol. 22, AGU, Washington, D. C., 1980.

Moldwin, M. B., L. Downward, H. K. Rassoul, R. Amin, and R. R. Anderson, A new model of the location of the plasmapause: CRRES results, J. Geophys. Res., 107(A11), 1339, doi:10.1029/2001JA009211, 2002.

Nishida, A., Formation of plasmapause, or magnetospheric plasma knee, by the combined action of magnetospheric convection and plasma escape from the tail, J. Geophys. Res., 71, 5669-5679, 1966.

Press, W. H., S. A. Teukolsky, W. T. Betterling, and B. P. Flannery, Numerical Recipes in C: The Art of Scientific Computing, Cambridge Univ. Press, New York, 1992.

Ridley, A. J., and M. W. Liemohn, A model-derived stormtime asymmetric ring current driven electric field description, J. Geophys. Res., 107(A8), 1151, doi:10.1029/2001JA000051, 2002.

Tverskaya, L. V., On the boundary of electron injection into the magnetosphere, Geomagn. Aeron., 26, 864-865, 1986.

Volland, H., Semiempirical models of magnetospheric electric fields, in Quantitative Modeling of Magnetospheric Processes, Geophys. Mongr. Ser., vol. 21, edited by W. P. Olson, AGU, Washington, D. C., 1979.

M. B. Moldwin, Institute of Geophysics and Planetary Physics, University of California, Los Angeles, CA 90095-1567, USA. (mmoldwin@) igpp.ucla.edu)

T. P. O’Brien, Space Science Department, Aerospace Corporation, M2260, P.O. Box 92957, El Segundo, CA 90009-92957, USA. (paul.obrien@ aero.org) 